\title{
Leveraging Public Health Emergency Informatics during the Fungal Infections Outbreak, Tennessee-2012
}

\author{
Rendi Murphree*1, 2, Paul Petersen 1 , Laina Stanford ${ }^{1}$, Jeff C. Sexton ${ }^{1}$, Tonya McKennley ${ }^{1}$, \\ James Milliken ${ }^{1}$, Tristan Victoroff ${ }^{1,2}$, Robert Newsad ${ }^{1,2}$ and Joseph Roth ${ }^{1,2}$
}

${ }^{1}$ Tennessee Department of Health, Nashville, TN, USA; ${ }^{2}$ Centers for Disease Control and Prevention, Atlanta, GA, USA

\section{Objective}

The purpose was to facilitate real-time information sharing and data visualization for incident management during the Fungal Infections Outbreak in Tennessee.

\section{Introduction}

Late in September 2012, the Tennessee Department of Health (TDH) identified a cluster of fungal infections following epidural injection of methylprednisolone acetate (MPA) from a single compounding pharmacy. This presented a public health imperative to contact, educate and monitor approximately 1,100 Tennessee residents who received injections from contaminated MPA lots that were shipped to three clinics in Tennessee. There was no precedent to accomplish this rapidly and efficiently. To accomplish this goal a secure, web-based application designed by TDH for emergency patient management was deployed. The purpose was to facilitate real-time information sharing and incident management data visualization.

\section{Methods}

The patient management application was designed by TDH and developed by a commercial vendor as part of the Tennessee Countermeasure Response Network (TNCRN). Patient demographic data were obtained from 1,100 patients who received injection(s) at one of three affected clinics. The data were managed by TDH in a relational Microsoft Access ${ }^{\circledR}$ database and also imported into TNCRN. Field staff used TNCRN to track patient locations (public health jurisdictions), document weekly patient consultations and symptom surveys, assign triage status, and document final disposition. Any patient who received a diagnostic lumbar puncture post injection was referred for full investigation.

\section{Results}

Within 24 hours of the decision to use TNCRN for this public health response more than 100 field staff were trained to use TNCRN via webinar. During the first six weeks of using TNCRN, 2,341 symptom surveys were conducted and 3,164 consultations documented. Using TNCRN, these data were easily shared among TDH and field staff, facilitating rapid communication among practitioners. As of 11 December 2012, 48 (41\%) of Tennessee's 116 confirmed case patients were first recognized by field staff and referred for investigation using TNCRN.

\section{Conclusions}

The use of TNCRN during this aggressive campaign to monitor MPA-exposed patients facilitated quick identification of potential case patients needing full investigation. Without real-time information sharing through TNCRN, communications among public health practitioners at state, regional, and local levels may have been delayed, inaccurate, fragmented, and inefficient. The use of the TNCRN in this outbreak response demonstrated that web-based information systems can be leveraged for emergency uses in public health practice.

\section{Keywords}

Tennessee; fungal infections outbreak; patient management; emergency informatics

\author{
${ }^{\star}$ Rendi Murphree \\ E-mail: zle9@cdc.gov
}

